\title{
O-minimal cohomology: finiteness and invariance results
}

\author{
Alessandro Berarducci* \& Antongiulio Fornasiero
}

May 26, 2007

\begin{abstract}
We prove that the cohomology groups of a definably compact set over an o-minimal expansion of a group are finitely generated and invariant under elementary extensions and expansions of the language. We also study the cohomology of the intersection of a definable decreasing family of definably compact sets under the additional assumption that the o-minimal structure expands a field.
\end{abstract}

\section{Contents}

1 Introduction . . . . . . . . . . . . . . . . 目

2 Topological preliminaries . . . . . . . . . . . 3

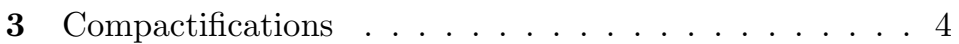

4 Cohomology of definable sets . . . . . . . . . .

5 Contractibility of cells . . . . . . . . . . . . . . 7

6 Cells with non-acyclic closure . . . . . . . . . . 9

7 Acyclic coverings . . . . . . . . . . . . . . 110

8 Finiteness results for cohomology . . . . . . . . . . 12

9 Elementary extensions and change of language . . . . 16

10 Definable families in expansions of fields . . . . . . . 177

Appendices . . . . . . . . . . . . . . . . . . .

A Derived functors $\ldots \ldots \ldots \ldots \ldots$. . . . . . . . . . .

B Flabby and soft sheaves . . . . . . . . . . . 23

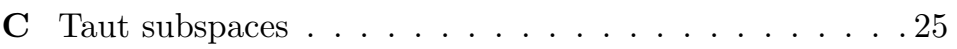

D Čech cohomology .................

\section{Introduction}

Delfs Delfs85 considered a sheaf cohomology theory for (abstract) semialgebraic sets over arbitrary closed fields and proved a semialgebraic version of homotopy invariance. In EdmJP05 this was generalized to definable sets

*Partially supported by the project: Geometría Real (GEOR) DGICYT MTM200502865 (2006-08) 
and maps over an o-minimal expansion of a group. If one further assumes that the o-minimal structure expands a field, then one can use the triangulation theorem to show that the cohomology groups of a definable set are finitely generated and invariant under both elementary extensions and expansions of the language. We prove that this continues to hold for arbitrary o-minimal expansions of groups, provided we restrict ourselves to definably compact sets.

Working without the field assumption entails various difficulties. To begin with one cannot make use of the apparatus of singular cohomology. So we work, following the above authors, with sheaf cohomology. More precisely, given a definable set $X \subset M^{n}$, the set of types $\widetilde{X}$ of $X$ with the "spectral topology" is quasi-compactification of $X$, and we define $\mathrm{H}^{i}(X ; \mathcal{F}):=\mathrm{H}^{i}(\widetilde{X} ; \mathcal{F})$, where $\mathcal{F}$ is a sheaf of Abelian groups on $\widetilde{X}$.

Now consider the case when $G$ is an Abelian group and $\mathcal{F}$ is the constant sheaf $G$ (i.e. the sheaf generated by the presheaf with constant value $G$ ). Assuming that $X$ is definably compact (i.e. closed and bounded) we prove that $\mathrm{H}^{i}(X ; G)$ is finitely generated and invariant under both elementary extensions $N \succ M$ (i.e. $\mathrm{H}^{i}(X ; G)=\mathrm{H}^{i}(X(N) ; G)$ ) and expansions of the language of $M$ (note that expanding the language leaves $X$ invariant but alters $\widetilde{X})$.

This would be easy to prove if $M$ expands a field. In fact in this case by the triangulation theorem $X$ is definably homeomorphic to the geometrical realization $|K|$ (in $M$ ) of a finite simplicial complex $K$, and a routine MayerVietoris argument (together with the acyclicity of simplexes) shows that $\mathrm{H}^{i}(X ; G) \cong \mathrm{H}^{i}(K ; G)$, where the latter is the $i$-th simplicial cohomology group of $K$.

If $M$ does not expand a field we do not have the triangulation theorem but we still have the cell decomposition theorem (see Dries98]). One could then be tempted to invoke the uniqueness theorem for cohomology functors satisfying the (appropriate form) of the Eilenberg-Steenrod axioms. However, despite the cell decomposition theorem, we have no guarantee that a definable set in an o-minimal expansion of a group is a sort of definable CWcomplex, so the uniqueness theorem does not apply. The problem is that in the definition of a $\mathrm{CW}$-complex one requires that the cells come equipped with an attaching map that extends continuously to the boundary, while for the o-minimal cells we do not have any such control of the boundary.

As standard references on sheaf cohomology and Čech cohomology we use God73] and Bred97. For the reader's convenience we give in the appendixes the relevant definitions and results. Sheaf cohomology is defined for arbitrary topological spaces, but many results are proved in the quoted texts under the additional assumption that the space (or the family of supports) is Hausdorff and paracompact. This is potentially a source of problems since, given a definable set $X$, the spectral space $\widetilde{X}$ associated to it is in general not Hausdorff. In some cases we can reduce to the compact Hausdorff 
situation using the fact that $\widetilde{X}$ has a continuous retraction onto a compact Hausdorff subspace $\widetilde{X}^{\text {max }}$ with the same cohomology groups (see CarrC83), but in some other cases it is more convenient to show that the proofs of the relevant results in God73 or Bred97] work with the Hausdorff hypothesis being replaced by normality (according to our convention normality does not imply Hausdorff). The latter approach has the advantage that one can do without the spectrality hypothesis. In particular it can be shown that for normal paracompact spaces (not assumed to be Hausdorff or spectral) sheaf cohomology coincides with Cech cohomology with coefficients in the given sheaf. This is reported in appendix $\mathrm{D}$, but we shall not really need it. Instead we do need some results connecting the cohomology of a subspace with the cohomology of its neighbourhoods. Such results have been proved in [Delfs85, Jones06, EdmJP05] in the spectral situation (see Corollary C.5), but they can also been established under more general hypothesis (Theorem C.4). As a corollary we obtain that $\mathrm{H}^{*}\left(\bigcap_{t>0} Y_{t}\right) \cong \lim _{t} \mathrm{H}^{*}\left(Y_{t}\right)$, whenever $\left(Y_{t} \mid t>0\right)$ is a definable decreasing family of definably compact sets $Y_{t}$ (Corollary C.6). We will also show (Theorem 10.3) that if the o-minimal structure $M$ expands a field, then $\lim _{t} \mathrm{H}^{*}\left(Y_{t}\right) \cong \mathrm{H}^{*}\left(Y_{t_{0}}\right)$ for all sufficiently small $t_{0}$, but we are not able to prove this fact without the field assumption.

\section{Topological preliminaries}

Let $X$ be a topological space. $X$ is normal if every pair of disjoint closed subsets of $X$ can be separated by open neighbourhoods. $X$ is paracompact if every open covering of $X$ has a locally finite refinement. Unlike other authors, in both definitions we do not require that $X$ be Hausdorff. We shall call a space $\mathbf{P} \mathbf{c N}$ if it is paracompact and normal. Note that a paracompact Hausdorff space is $\mathrm{PcN}$.

Let us recall that a quasi-compact space is a topological space $X$ in which every open covering has a finite refinement, or equivalently every family of closed sets with the finite intersection property has a non-empty intersection. So a compact space is an Hausdorff quasi-compact space.

Note that a quasi-compact space is a fortiori paracompact. Moreover, it is a well-known fact that a in a $\mathrm{PcN}$ space $X$ every open covering $\mathcal{U}$ admits a shrinking $\mathcal{V}$ : i.e., $\mathcal{V}$ is an open covering of $X$, and for every $V \in \mathcal{V}$ there exists $U \in \mathcal{U}$ containing $\bar{V}$, the closure of $V$.

A spectral space is a quasi-compact space having a basis of quasicompact open sets stable under finite intersections and such that every irreducible closed set is the closure of a unique point. The prime spectrum of a commutative ring with its Zariski topology is an example of a spectral space. Another example is the set of prime filters of a lattice (see CarrC83). The set of $n$-types (ultrafilters of definable sets) of a first order topological structure $M$ in the sense of [Pillay87] can also be endowed with a spectral 
topology (see [Pillay88]). In particular if $M$ is a real closed field one obtains in this way the real spectrum of the polynomial ring $M\left[x_{1}, \ldots, x_{n}\right]$ (see [CosteR82]).

\section{Compactifications}

In this section we discuss a variant of the Wallman (or Stone-Čech) compactification of a normal topological space. The variant depends on the particular choice of a basis of open sets.

Definition 3.1. Given a topological space $X$ with a fixed basis of open sets $\mathcal{U}$ (that we always assume closed under finite intersections) we can define a spectral space $\tilde{X}=\widetilde{(X, \mathcal{U})}$ (depending on $\mathcal{U}$ ) as follows. A constructible set is a boolean combination of basic open sets $U \in \mathcal{U}$. Let $\widetilde{X}$ be the set of ultrafilters of constructible sets (i.e. maximal families of constructible sets closed under finite intersections and not containing the empty set). For $b \subset X$ constructible, let $\tilde{b}=\{p \in \widetilde{X} \mid b \in p\}$. So $p \in \tilde{b} \Longleftrightarrow b \in p$. The spectral topology on $\tilde{X}$ is defined as follows. As a basis of open sets of $\widetilde{X}$ we take the sets of the form $\tilde{b}$ with $b$ an open constructible subset of $X$.

Lemma 3.2. $\widetilde{X}$ is a spectral space.

Proof. To prove that $\widetilde{X}$ is quasi-compact consider a family $\left\{C_{i} \mid i \in I\right\}$ of closed sets $C_{i} \subset \widetilde{X}$ with the finite intersection property. We must prove that $\bigcap_{i \in I} C_{i}$ is non-empty. Without loss of generality we can assume that $\left\{C_{i} \mid i \in I\right\}$ is closed under finite intersections. Let $x \in \widetilde{X}$ be an ultrafilter containing all the closed constructible sets $b$ with $\widetilde{b} \supset C_{i}$ for some $i$. Then $x \in \bigcap_{i} C_{i}$, so $\widetilde{X}$ is quasi-compact. The same argument shows that the sets $\widetilde{b}$ with $b$ constructible are quasi-compact. So the sets $\widetilde{b}$, with $b$ open and constructible, form a basis of quasi-compact open sets of $\widetilde{X}$ stable under finite intersections. To finish the proof we must show that given an irreducible closed set $C$ of $\tilde{X}$, there is a unique point $x \in C$ with $C=C l(x)$. To this aim, let $x$ be an ultrafilter of constructible sets containing all the closed constructible sets $b$ with $\widetilde{b} \supset C$ and the complements of the closed constructible sets $c$ such that $\widetilde{c} \cap C$ is a proper subset of $C$ (this family has the finite intersection property by the irreducibility of $C$ ). Then clearly $C l(x)=C$. To prove that $x$ is unique, suppose $C l(x)=C l(y)$. Then $x$ and $y$ contain the same closed constructible sets. But the closed constructible sets generate the boolean algebra of all the constructible sets. So $x$ and $y$ must contain the same constructible sets, and are therefore equal.

Remark 3.3. Note that a point $x \in \tilde{X}$ is closed if and only if $x$ contains a maximal family of closed constructible sets with the finite intersection property. So by Zorn's lemma every closed subset $C$ of $\widetilde{X}$ contains a closed point. 
For $x \in X$ let

$$
\langle x\rangle:=\{b \mid x \in b\} \in \tilde{X} .
$$

Since a constructible set $b$ is empty if and only if $\widetilde{b}$ is empty, the map

$$
\langle\rangle: X \rightarrow \tilde{X}
$$

has dense image. Moreover this map is injective whenever $X$ is a $T_{1}$-space. So in this case, identifying $x$ with $\langle x\rangle$, we have $X \subset \widetilde{X}$, and it is easy to see that the original topology on $X$ coincides with the topology induced by $\widetilde{X}$ (use the fact that for $A$ constructible, $\widetilde{A} \cap X=A$ ). Therefore:

Lemma 3.4. If $X$ is $T_{1}$, then for every open basis $\mathcal{U}$ of $X, \widetilde{(X, \mathcal{U})}$ is a quasi-compactification of $X$.

We say that $(X, \mathcal{U})$ is constructibly normal if any pair of disjoint constructible open sets can be separated by closed constructible sets.

Lemma 3.5. If $(X, \mathcal{U})$ is constructibly normal, then $\tilde{X}$ is normal (not necessarily Hausdorff).

Proof. Indeed given two disjoint closed subsets $A$ and $B$ of $\widetilde{X}$, by quasicompactness there are disjoint closed constructible sets $A^{\prime}, B^{\prime}$ in $X$ with $\widetilde{A^{\prime}} \supset A$ and $\widetilde{B^{\prime}} \supset B$. By the assumption $A^{\prime}, B^{\prime}$ can be separated by disjoint open constructible sets $U \supset A^{\prime}$ and $V \supset B^{\prime}$. So $\widetilde{U}$ and $\widetilde{V}$ are open sets separating $A, B$.

In a normal spectral space $Y$, the subset $Y^{\text {max }}$ of the closed points of $Y$ is compact Hausdorff (see CarrC83). Also note that, if $x$ is a closed point of $X$ and the singleton $\{x\}$ is constructible, then $\langle x\rangle$ is a closed point of $\widetilde{X}$. So we have:

Lemma 3.6. If $(X, \mathcal{U})$ is constructibly normal and the points of $X$ are closed and constructible, then $\widetilde{X}^{\max }$ is a compactification of $X$, namely it is a compact Hausdorff space containing $X$ as a dense subspace.

If $X$ is a normal Hausdorff space, and we take as a basis of $X$ the family $\mathcal{U}$ of all its open subsets, then $\widetilde{X}^{\max }$ is the Wallman compactification of $X$ [Wallm38]. For normal Hausdorff spaces it coincides with the Stone-Čech compactification (see Eng89, Thm. 3.6.22]).

Example 3.7. Consider the space $\mathbb{Q}$ with a basis $\mathcal{U}$ of open sets given by the open intervals $(a, b) \subset \mathbb{Q}$, where we allow $a=-\infty$ or $b=+\infty$. Then

$$
\widetilde{(\mathbb{Q}, \mathcal{U})}=\left\{a^{-}, a^{+}\right\}_{a \in \mathbb{Q}} \cup \mathbb{R} \cup\{ \pm \infty\}
$$

where: $a^{+}$is the unique ultrafilter containing all sets of the form $(a, b)$ with $b>a$; similarly $a^{-}$contains all sets $(b, a)$ with $b<a ;+\infty$ is the ultrafilter 
containing $(a, \infty)$ for all $a \in \mathbb{Q}$; finally $-\infty$ contains $(-\infty, a)$ with $a \in \mathbb{Q}$. We have $C l\left(a^{-}\right)=\left\{a^{-}, a\right\}$ and $C l\left(a^{+}\right)=\left\{a^{+}, a\right\}$. The set of closed points is

$$
\widetilde{(\mathbb{Q}, \mathcal{U})}^{\max }=\mathbb{R} \cup\{ \pm \infty\}
$$

a two point-compactification of $\mathbb{R}$.

Example 3.8. Consider the space $\mathbb{Q}$ with a basis $\mathcal{U}$ of open sets given by the bounded open intervals $(a, b) \subset \mathbb{Q}$. Then

$$
\widetilde{(\mathbb{Q}, \mathcal{U}}^{\max }=\mathbb{R} \cup\{\infty\}
$$

the one-point compactification of $\mathbb{R}$.

\section{Cohomology of definable sets}

Let $M=(M,<, \ldots)$ be an o-minimal structure (cf. [Dries98]) expanding a dense linear order $(M,<)$. For instance $M$ may be a divisible ordered group or a real closed field. We put on $M$ the topology generated by the open intervals and on $M^{n}$ the product topology. If $X$ is a subset of $M^{n}$ we put on $X$ the induced topology from $M^{n}$ unless otherwise stated. By a definable set we mean a first-order definable (with parameters) subset of $M^{n}$ for some $n$. For instance if $M$ is a real closed field, the definable sets are the semialgebraic sets. Let $X \subset M^{n}$ be a definable set. Let $\mathcal{U}$ be the basis of $X$ consisting of the open definable subsets of $X$. Define $\widetilde{X}=\widetilde{(X, \mathcal{U})}$ as in Definition 3.1. Note that, by the o-minimality assumption, the definable sets coincide with the constructible ones, namely every definable set is a boolean combination of open definable sets. It follows that $\widetilde{X}$ is the set of types of $X$ (a type $p \in \widetilde{X}$ can be identified with an ultrafilter of definable sets such that $X \in p$ ) endowed with the spectral topology: as a basis of open sets we take the sets of the form $\widetilde{U}$ with $U$ a definable open subset of $X$. Since $X$ is Hausdorff, by Lemma 3.4 $\widetilde{X}$ is a quasi-compactification of $X$, in general not Hausdorff.

Let us now make the further assumption that $M$ is an o-minimal expansion of an ordered group. In this case one can use the $M$-valued metric $|x-y|$ to show that every definable set $X$ is definably normal (any pair of disjoint definable closed sets can be separated by definable open sets). Therefore in this case $\widetilde{X}$ is normal (not necessarily Hausdorff), and the subspace $\widetilde{X}^{\text {max }}$ of its closed points is compact Hausdorff (and contains $X$ as a topological subspace).

Given a definable set $X \subset M^{n}$ and a sheaf of Abelian groups $\mathcal{F}$ on $\tilde{X}$, we define, following Delfs85, Jones06, EdmJP05]:

$$
\mathrm{H}^{i}(X ; \mathcal{F}):=\mathrm{H}^{i}(\widetilde{X} ; \mathcal{F})
$$


see Definition B.1. Equivalently one can work with sheaves directly on $X$ rather than $\widetilde{X}$ by considering $X$ not as a topological space but as a site in the sense of Groethendieck (see CarrC83, $\S 1.3]$ ). If $A$ is a definable subset of $X$, we write $\mathrm{H}^{*}(A ; \mathcal{F})$ for $\mathrm{H}^{*}(\widetilde{A} ; \mathcal{F}\lceil\widetilde{A})$.

If $f: X \rightarrow Y$ is a definable function, then $f$ induces a function $\tilde{f}: \widetilde{X} \rightarrow \widetilde{Y}$ by $f(p)=\left\{Z \mid f^{-1}(Z) \in p\right\}$ where $Z$ ranges over the definable subsets of $Y$ and we identify $p$ with an ultrafilter of definable sets. We have $\widetilde{f(X)}=\widetilde{f}(\widetilde{X})$ and $\widetilde{f}^{-1}(\widetilde{Z})=\widetilde{f^{-1}(Z)}$. It follows that if $f$ is continuous, $\widetilde{f}$ is continuous. So if $f$ is an homeomorphism, then $\tilde{f}$ is an homeomorphism.

If $G$ is an Abelian group and $X$ is a topological space, the constant sheaf on $X$ with stalk $G$ will also be denoted $G$.

We recall that a definable homotopy between two definable functions $f: X \rightarrow Y$ and $g: X \rightarrow Y$ is a definable continuous function $F: I \times X \rightarrow Y$, where $I=[a, b]$ is some closed bounded interval in $M$, such that $F(a, x)=$ $f(x)$ and $F(b, x)=g(x)$ for every $x \in X$. Note that $F$ induces a map $\widetilde{F}: \widetilde{I \times X} \rightarrow \widetilde{Y}$, but in general $\widetilde{I \times X} \neq \widetilde{I} \times \widetilde{X}$, so we cannot consider $\widetilde{F}$ as a sort of "homotopy" parametrised by $\widetilde{I}$. Nevertheless we have the following definable version of the homotopy axiom:

Fact 4.1. (Delfs85, Jones06, EdmJP05]) If $X, Y$ are definable sets and $f, g: X \rightarrow Y$ are definably homotopic definable maps, then $\tilde{f}, \widetilde{g}: \widetilde{X} \rightarrow \widetilde{Y}$ (although not necessarily homotopic) induce the same homomorphism in cohomology, namely for every Abelian group $G$ we have

$$
f^{*}=g^{*}: \mathrm{H}^{i}(Y ; G) \rightarrow \mathrm{H}^{i}(X ; G) .
$$

Similarly for maps $f, g:(X, A) \rightarrow(Y, B)$ of pairs.

This has been proved by Delfs85 in the semialgebraic case (i.e. when $M$ is a real closed field and $X, Y, f, g$ are semialgebraic). Jones [Jones06. extended it to the case of definable sets and maps in an o-minimal expansion $M$ of an ordered field. In EdmJP05 it is shown that it suffices that $M$ is an o-minimal expansion of an ordered group. All proofs make use of the following lemma:

Lemma 4.2. (Delfs85, Prop. 4.7]) Let $I \subset M$ be a closed and bounded interval $[a, b]$. Then $\mathrm{H}^{p}(I ; G)=0$ for all $p>0$ and every Abelian group $G$.

Indeed it is easy to see the lemma holds for an arbitrary interval $I$, not necessarily closed and bounded (note that in any case $\widetilde{I}$ is quasi-compact).

\section{Contractibility of cells}

Let $M$ be an o-minimal expansion of a group. 
Lemma 5.1. Let I be a bounded interval in $M$ (closed, half-closed, or open). Then $I$ is definably contractible to a point.

Proof. Let us consider the case $I=(a, b)$. Given $0<t \leq \frac{b-a}{2}$, there is a (unique) definable continuous function $f_{t}:(a, b) \rightarrow(a, b)$ such that $f_{t}$ is the identity on $[a+t, b-t]$ and it is constant on both $[a, a+t]$ and $[b-t, b]$, with values $a+t$ and $b-t$ respectively (so the image of $f_{t}$ is $[a+t, b-t]$ ). Define $f_{0}=f$. Then $\left(f_{t}\right)_{0 \leq t \leq \frac{b-a}{2}}$ is a deformation retract of $(a, b)$ to the point $\frac{a+b}{2}$. The other cases are similar.

We will employ the following notation: given $B \subseteq M^{n-1}$ and $f, g: B \rightarrow M$,

$$
\begin{aligned}
(f, g)_{B} & :=\left\{(x, y) \in M^{n-1} \times M: x \in B \& f(x)<y<g(x)\right\}, \\
{[f, g]_{B} } & :=\left\{(x, y) \in M^{n-1} \times M: x \in B \& f(x) \leq y \leq g(x)\right\}, \\
\Gamma(f) & :=\left\{(x, y) \in M^{n-1} \times M: x \in B \& f(x)=y\right\}, \text { the graph of } f .
\end{aligned}
$$

Lemma 5.2. If $C$ is a bounded cell of dimension $m>0$ in $M^{n}$ then there is a deformation retract of $C$ onto a cell of strictly lower dimension. So by induction every bounded cell is definably contractible to a point.

Proof. If $C$ is the graph of a function we can reason by induction on the dimension of the ambient space. So the only interesting case is when $m>1$ and $C=(f, g)_{B}$. Let $h=\frac{f+g}{2}$. We will define a deformation retract from $C$ to $\Gamma(h)$. We can assume that $h$ is a constant function, since we can reduce to this case by a definable homeomorphism which fixes all but the last coordinate (just take any constant function $h_{1}: B \rightarrow M$, and define $f_{1}, g_{1}$ so that they differ from $h_{1}$ by the same amount in which $f, g$ differ from $h$ ). Since $C$ is bounded, there are constants $a, b \in M$ such that $h$ is the constant function $\frac{a+b}{2}$ and $(f, g)_{B} \subset B \times(a, b)$. By (the proof of) Lemma 5.1 there is deformation retract of $(a, b)$ onto $\left\{\frac{a+b}{2}\right\}$, which induces a deformation retract of $B \times(a, b)$ onto $B \times\left\{\frac{a+b}{2}\right\}$, namely onto the graph of $h$.

By Fact 4.1 we obtain:

Corollary 5.3. If $C$ is a bounded cell of dimension $m$ in $M^{n}$ then $\mathrm{H}^{p}(C ; G)=$ 0 for all $p>0$ and every Abelian group $G$.

If we generalize slightly the definition of definable homotopy and allow the parameter of a homotopy to vary in the interval $[-\infty,+\infty]$, we get that Fact 4.1 is still true, and therefore in Lemmata 5.1 and 5.2 we can drop the "boundedness" hypothesis. Thus, Corollary 5.3 is true also for unbounded cells. 


\section{Cells with non-acyclic closure}

Let $M$ be an o-minimal expansion of a group and let $X \subset M^{n}$ be a definable set. We will prove (Theorem 8.5) that the cohomology groups $\mathrm{H}^{p}(X ; G)$ of $X$ are finitely generated. An important special case is when $X$ is the closure $\bar{C}$ of a bounded cell $C$. One may be tempted to conjecture that $\mathrm{H}^{i}(\bar{C} ; G)=0$ in dimension $i>0$, but Example 6.1 shows that in general this is false. Indeed similar examples show that $\mathrm{H}^{1}(\bar{C} ; G)$ can have arbitrarily large finite rank.

Example 6.1. Let $M=(\mathbb{R},<,+, \cdot)$. There is a bounded cell $C$ of dimension 2 in $\mathbb{R}^{4}$ whose closure $\bar{C}$ has an "hole", namely it is definably homotopic to a circle.

Before giving the example, let us observe that in Figure 1 (an annulus with a ray removed) we have a space homeomorphic to a disk whose closure is homotopic to a circle. However the space of Figure 1 is not a cell in the sense of o-minimal cell decompositions. So we have to proceed differently.

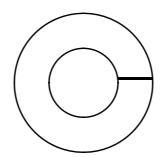

As a preliminary step we show that there is a two-dimensional cell $D$ in $\mathbb{R}^{3}$ which is homeomorphic to an open disk minus a ray via an homeomorphism which extends to the closures. An example is the cell $D$ depicted on the top-right part of Figure 2 .

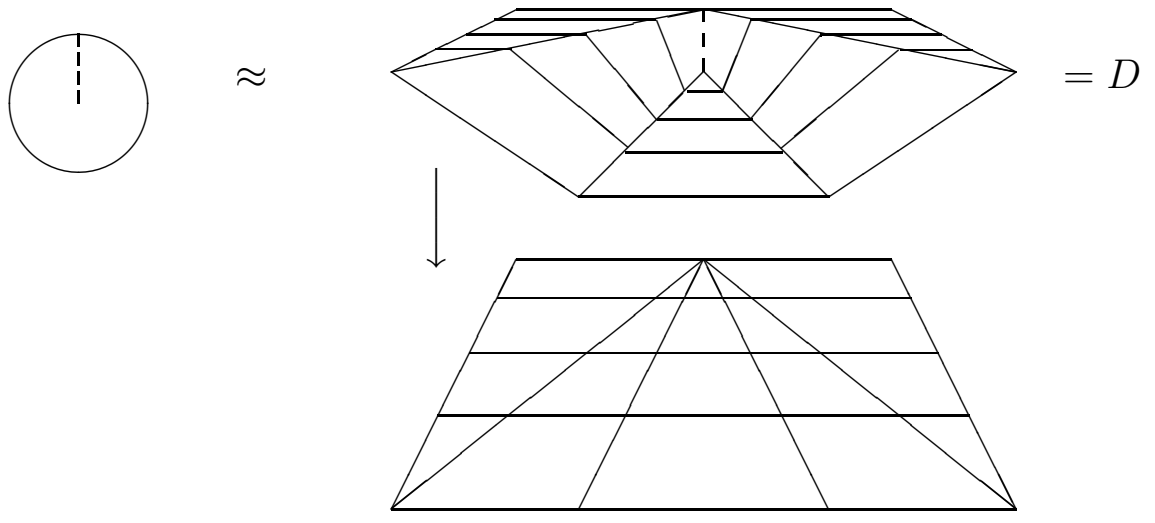

Figure 2. A two-dimensional cell in $\mathbb{R}^{3}$

Figure 2 must be interpreted as follows. The projection of $D$ on the first two coordinates is the open square on the bottom-right part of Figure 2, with the five triangles partitioning the square corresponding to the five regions in $D$. So $D$ is the graph of a function $f$ from the square to $\mathbb{R}$. $D$ is 
homeomorphic to an open disk with a ray removed via an homeomorphism that extends to the closures and sends the ray of the disk to the vertical dashed line $I \subset \partial D$ depicted in the Figure. The three triangular regions of the cell $D$ are level sets of $f$, with the central triangle being in a lower level, and the two top triangles being on a common higher level.

We are now ready to describe the cell $C$ of Example 6.1. The idea is the following. Given a disk without a ray as in Figure 2, we can "bend" it going in a higher dimension, so as to create a "hole" (as in Figure 1) by separating the two sides of the middle part of the ray. More precisely, consider a definable continuous function $g: D \rightarrow \mathbb{R}$ with the following properties: 1) $g$ takes non-zero values only for points $x \in D$ sufficiently close to the middle point $x_{0}$ of the dashed segment $\left.I \subset \partial D ; 2\right)$ if $g(x)>0$, then $x$ is on the "left hand side" of $I$ and $g(x) \rightarrow 1$ for $x \rightarrow x_{0}$ from the left; 3$)$ if $g(x)<0$, then $x$ is on the "right-hand-side" of $I$ and $g(x) \rightarrow-1$ for $x \rightarrow x_{0}$ from the right. Let $C$ be the graph of $g$. Then $H^{1}(\bar{C} ; G) \neq 0$.

Remark 6.2. It is possible to show that $\mathrm{H}^{1}(X ; G)$ can have arbitrarily high rank even for a definable set $X$ that can be decomposed as a disjoint union of only two bounded cells. Therefore, unlike the case of triangulations, the abstract structure of a cell decomposition (namely the dimensions and the adjacency relation between cells) by no means determines the cohomology of a definable set. It is not however excluded that by a refined version of the cell decomposition theorem one could obtain decompositions that do determine the cohomology groups.

\section{$7 \quad$ Acyclic coverings}

In this section we give a sufficient condition, not based on deformation retracts, to prove that an inclusion $X \subset Y$ of topological spaces induces an isomorphism in cohomology (see Lemma 7.5).

Given an open cover $\mathcal{U}=\left\{U_{i} \mid i \in I\right\}$ of a topological space $X$, and a subset $J \subset I$, we write $U_{J}$ for the intersection $\bigcap_{i \in J} U_{i}$. The following theorem of Leray says that, given an "acyclic covering", the cohomology of a sheaf can be computed as the Cech cohomology of the covering (cf. Appendix D).

Fact 7.1. ([Bred97, Thm. 4.13, p. 193], God73, §5.4, p. 213]) Let $\mathcal{F}$ be a sheaf on a topological space $X$ and let $\mathcal{U}=\left\{U_{i} \mid i \in I\right\}$ be an open covering of $X$ having the property that $\mathrm{H}^{p}\left(U_{J} ; \mathcal{F}\right)=0$ for every $p>0$ and every finite $J \subset I$. Then the canonical homomorphism $\check{\mathrm{H}}^{*}(\mathcal{U} ; \mathcal{F}) \rightarrow \mathrm{H}^{*}(X ; \mathcal{F})$ is an isomorphism.

If moreover we assume that $\mathcal{F}$ is the constant sheaf $G$ and $\mathrm{H}^{0}\left(U_{J} ; \mathcal{F}\right)=G$ for every $J$ with $U_{J}$ non-empty, then from the definition of Cech cohomology it follows that $\check{\mathrm{H}}^{*}(\mathcal{U} ; G)$ coincides with the $i$-th simplicial cohomology group with coefficients in $G$ of the nerve $N(\mathcal{U})$ of $\mathcal{U}$. So we have: 
Corollary 7.2. If there exists a finite open cover $\mathcal{U}=\left\{U_{i} \mid i \in I\right\}$ of $X$ with $\mathrm{H}^{p}\left(U_{J} ; G\right)=0$ for every $p>0$ and $\mathrm{H}^{0}\left(U_{J} ; G\right)=G$ for every finite $J \subset I$ with $U_{J}$ non-empty, then $\mathrm{H}^{i}(X ; G)$ is isomorphic to the $i$-th simplicial cohomology group of the nerve $N(\mathcal{U})$ of the covering, so in particular it is finitely generated.

Unfortunately we do not know the answer to the following:

Question 7.3. Let $X$ be a definably compact set in an o-minimal expansion $M$ of a group. Does $\widetilde{X}$ have a cover as in Corollary 7.9?

The answer is positive if $M$ expands a field, as a simple application of the triangulation theorem shows.

Remark 7.4. Let $f: X \rightarrow Y$ be a continuous function, let $\mathcal{V}=\left\{V_{i} \mid i \in I\right\}$ be an (indexed) open cover of $Y$ and consider the (indexed) open cover $f^{-1}(\mathcal{V}):=\left\{f^{-1}\left(V_{i}\right) \mid i \in I\right\}$ of $X$. It follows easily from the definition of the induced homomorphism $\check{f}$ in Čech cohomology (see [EilS52, IX, §4] or Bred97, III.4.1.4]) that there is a commutative diagram:

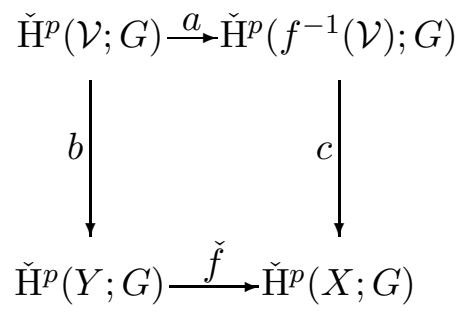

where $b, c$ are the natural morphisms (see God73]) and $a$ is induced by the simplicial map on the nerves of the indexed coverings sending $f^{-1}\left(V_{i}\right)$ to $V_{i}$.

Lemma 7.5. Let $X \subset Y$ be definable sets. Suppose that there are coverings $\mathcal{U}=\left\{U_{i} \mid i \in I\right\}$ of $X$ and $\mathcal{V}=\left\{V_{i} \mid i \in I\right\}$ of $Y$ indexed by the same finite set $I$ such that:

1. $U_{i} \subset V_{i}$ for all $i, j \in I$.

2. For all finite $F \subset I, U_{F}$ is non-empty iff $V_{F}$ is non-empty (i.e. the natural map among the nerves of the coverings is an isomorphism).

3. For each finite $F \subset I$ the sets $U_{F}$ and $V_{F}$ are either empty or connected, and for all $q>0, \mathrm{H}^{q}\left(U_{F} ; G\right)=\mathrm{H}^{q}\left(V_{F} ; G\right)=0$.

Then the inclusion map $X \subset Y$ induces an isomorphism $\mathrm{H}^{*}(Y ; G) \rightarrow$ $\mathrm{H}^{*}(X ; G)$ for any Abelian group $G$.

Note that we do not require that $V_{i} \cap X=U_{i}$. 
Proof. We are going to apply Remark 7.4 to the case when $X \subset Y$ and $f$ is the inclusion map. In this case $f^{-1}(\mathcal{V})=\mathcal{V} \cap X$ (by definition). Consider the following commutative diagram, where $a, b, c$ are as in Remark 7.4, $d$ is induced by the natural simplicial isomorphism on the nerves of the coverings, $e$ is the natural morphism form the Čech cohomology of a covering to the Čech cohomology of the space, and $p, q$ are the natural maps from Čech to sheaf cohomology.

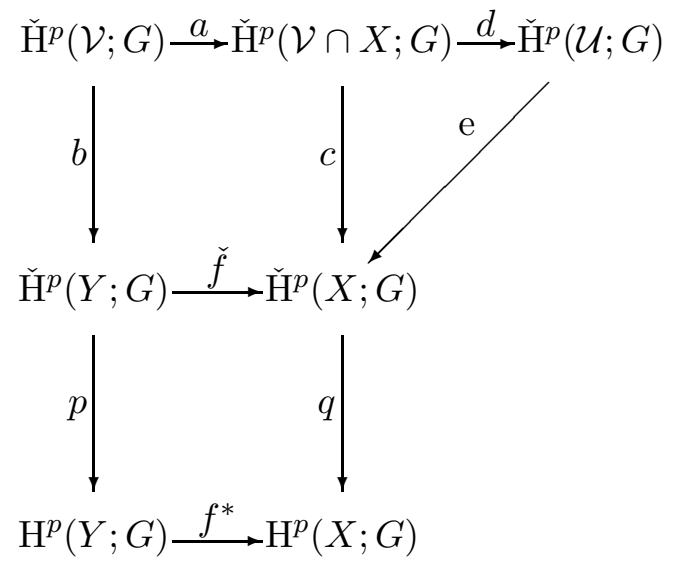

By our assumptions on the coverings, $a$ and $d$ are isomorphisms. By Fact $7.1 p \circ b$ and $q \circ e$ are isomorphisms. So $f^{*}: \mathrm{H}^{*}(Y ; G) \rightarrow \mathrm{H}^{*}(X ; G)$ is an isomorphism.

\section{Finiteness results for cohomology}

As usual let $M$ be an o-minimal expansion of group. Given a definably compact set $X$ we want to prove that $\mathrm{H}^{i}(X ; G)$ is finitely generated for every $i$ and every Abelian group $G$. An important special case is when $X$ is the closure $\bar{C}$ of a bounded cell $C$. We will show (as a consequence of Corollary 8.4) that there is a point $a \in C$ such that $\mathrm{H}^{p}(\bar{C} \backslash\{a\} ; G) \cong \mathrm{H}^{p}(\partial C ; G)$, where $\partial C:=\bar{C} \backslash C$ is the boundary of $C$. Granted this, since $\partial C$ has smaller dimension than $\bar{C}$, by induction on the dimension we can assume that the cohomology groups of $\partial C$ are finitely generated and carry on with the inductive proof. At first sight the fact that $\mathrm{H}^{p}(\bar{C} \backslash\{a\} ; G) \cong \mathrm{H}^{p}(\partial C ; G)$ looks rather intuitive: one may even be tempted to conjecture that $\partial C$ is a definable deformation retract of $\bar{C} \backslash\{a\}$ (as it would be the case for the cells of a CW-complex), or at least that these two spaces are definably homotopy equivalent. However we are not able to prove this. We proceed instead in a different manner, with the role of definable deformation retracts being taken by Lemma 7.5. There is however a further complication. We are not able to apply Lemma 7.5 directly to the pair of sets $(X, Y)=(\bar{C} \backslash\{a\}, \partial C)$, 
but only to pairs of sets of the form $\left(C \backslash\{a\}, C \backslash C_{t}\right)$, where $\left(C_{t}\right)_{t>0}$ is a suitable definable collection of sets with $\bigcup_{t>0} C_{t}=C$, and consequently $\bigcap_{t>0}\left(\bar{C} \backslash C_{t}\right)=\partial C$ (the singleton $\{a\}$ is one of the $\left.C_{t}\right)$. So we first prove $\mathrm{H}^{p}(C \backslash\{a\}) \cong \mathrm{H}^{p}\left(C \backslash C_{t}\right)$. Then we deduce $\mathrm{H}^{p}(\bar{C} \backslash\{a\}) \cong \mathrm{H}^{p}\left(\bar{C} \backslash C_{t}\right)$ by the excision theorem. Finally, with the help of Corollary C.6, we let $t \rightarrow 0$ to obtain $\mathrm{H}^{p}(\bar{C} \backslash\{a\}) \cong \mathrm{H}^{p}(\partial C)$. This is the idea. Let us now come to the details.

Lemma 8.1. Let $C \subset M^{n}$ be a bounded cell of dimension $m$. There is a definable family $\left\{C_{t} \mid t>0\right\}$ of definably compact sets $C_{t} \subset C$ such that:

1. $C=\bigcup_{t>0} C_{t}$.

2. If $0<t^{\prime}<t$, then $C_{t} \subset C_{t^{\prime}}$ and the inclusion $C \backslash C_{t^{\prime}} \subset C \backslash C_{t}$ induces an isomorphism $\mathrm{H}^{p}\left(C \backslash C_{t} ; G\right) \rightarrow \mathrm{H}^{p}\left(C \backslash C_{t^{\prime}} ; G\right)$.

3. For every Abelian group $G, C \backslash C_{t}$ has the same cohomology groups of an $m-1$ dimensional sphere, namely $\mathrm{H}^{p}\left(C \backslash C_{t} ; G\right)=0$ for $p \notin$ $\{0, m-1\}$, and $\mathrm{H}^{p}\left(C \backslash C_{t} ; G\right)=G$ for $p \in\{0, m-1\}$.

Note that the result would be obvious if $M$ expands a field, since in that case $C$ is definably homeomorphic to an open ball, and each open ball is the increasing union of its concentric closed sub-balls.

Proof. We define $C_{t}$ as follows.

1. If $n=1$ and $C=(a, b)$, then $C_{t}=\left[a+\gamma_{t}, b-\gamma_{t}\right]$ where $\gamma_{t}=\min \left\{\frac{a+b}{2}, t\right\}$ (so $C_{t}$ is non-empty).

2. If $n=1$ and $C$ is a singleton in $M, C_{t}=C$.

3. Let $n>1$ and $C=\Gamma(f)$, where $f: B \rightarrow M$. By induction $B_{t}$ is defined and we set $C_{t}=\Gamma\left(f\left\lceil B_{t}\right)\right.$.

4. Let $n>1$ and $C=(f, g)_{B}$. By induction $B_{t}$ is defined. We put $C_{t}=\left[f+\gamma_{t}, g-\gamma_{t}\right]_{B_{t}}$, where $\gamma_{t}:=\min \left(\frac{f-g}{2}, t\right)$.

With this definition we have:

Claim 1. For each $t>0$ there is a covering $\mathcal{U}=\left\{U_{i} \mid i \in I\right\}$ of $C \backslash C_{t}$ such that:

1. The index set $I$ is the family of the closed faces of an m-dimensional cube, where $m=\operatorname{dim}(C)$. $(S o|I|=2 m)$.

2. If $F \subset I$, then $U_{F}:=\bigcap_{i \in F} U_{i}$ is either empty or a cell. (So in particular $\mathrm{H}^{p}\left(U_{F} ; G\right)=0$ for all $p>0$ and, if $U_{F} \neq \emptyset, \mathrm{H}^{0}\left(U_{F} ; G\right)=G$.)

3. For $F \subset I, U_{F} \neq \emptyset$ iff the faces of the cubes belonging to $F$ have a non-empty intersections. (So the nerve of $\mathcal{U}$ is isomorphic to the nerve of a covering of an $m$-cube by its closed faces.) 
For example for $m=2$ we have four open sets $U_{i}$ which intersect each other as in Figure 3. Note that the claim implies, by Corollary 7.2, that $C \backslash C_{t}$ has the same cohomology groups of an $m-1$ dimensional sphere. To prove the claim we define $\mathcal{U}$ by induction on the dimension $n$ of the ambient space. We distinguish four cases according to the definition of $C_{t}$.

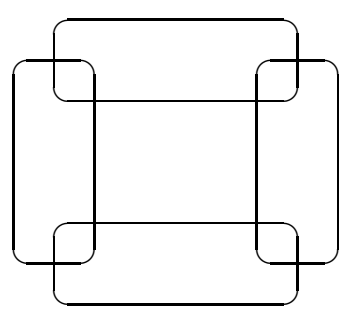

Figure 3.

1. If $n=1$ and $C$ is a singleton, then $\mathcal{U}$ is the covering consisting of one open set (given by the whole space $C$ ).

2. If $n=1$ and $C=(a, b)$, then $C \backslash C_{t}$ is the union of the two open subsets $\left(a, a+\gamma_{t}\right)$ and $\left(b-\gamma_{t}, b\right)$, and we define $\mathcal{U}$ as the covering consisting of these two sets.

3. Let $n>1$ and $C=\Gamma(f)$, where $f: B \rightarrow M$. By induction we have a covering $\mathcal{V}$ of $B \backslash B_{t}$ with the stated properties, and we define $\mathcal{U}$ to be the covering of $C \backslash C_{t}$ induced by the natural homeomorphism between the graph of $f$ and its domain.

4. Let $n>1$ and $C=(f, g)_{B}$. By definition $C_{t}=\left(f+\gamma_{t}, g-\gamma_{t}\right)_{B_{t}}$. By induction $B \backslash B_{t}$ has a covering $\mathcal{V}=\left\{V_{j} \mid j \in J\right\}$ with the stated properties, where $J$ is the set of closed faces of the cube $[0,1]^{m-1}$. Define a covering $\mathcal{U}=\left\{U_{i} \mid i \in I\right\}$ of $C \backslash C_{t}$ as follows. As index set $I$ we take the closed faces of the cube $[0,1]^{m}$. Thus $|I|=|J|+2$, the two extra faces corresponding to the "top" and "bottom" face of $[0,1]^{m}$. We associate to the top face the open set $\left(g-\gamma_{t}, g\right)_{B} \subset C \backslash C_{t}$ and to the bottom face the open set $\left(f, f+\gamma_{t}\right)_{B} \subset C \backslash C_{t}$. The other open sets of the covering are the preimages of the sets $V_{j}$ under the projection $M^{n} \rightarrow M^{n-1}$. This defines a covering of $C \backslash C_{t}$ with the stated properties.

It remains to show that the inclusion map $C \backslash C_{t^{\prime}} \subset C \backslash C_{t}$ induces an isomorphism $\mathrm{H}^{p}\left(C \backslash C_{t} ; G\right) \rightarrow \mathrm{H}^{p}\left(C \backslash C_{t^{\prime}} ; G\right)$. To this aim it suffices to observe that by (the proof of) Claim 1 there are coverings $\mathcal{U}$ of $C \backslash C_{t^{\prime}}$ and $\mathcal{V}$ of $C \backslash C_{t}$ satisfying the assumptions of Lemma 7.5.

Lemma 8.2. Let $X$ be a definably compact set, $C$ be a cell of maximal dimension in $X$, and $G$ be an Abelian group. Then for each $0<t^{\prime}<t$ the inclusion map $X \backslash C_{t^{\prime}} \subset X \backslash C_{t}$ induces an isomorphism

$$
\mathrm{H}^{*}\left(X \backslash C_{t} ; G\right) \cong \mathrm{H}^{*}\left(X \backslash C_{t^{\prime}} ; G\right) .
$$

Proof. By the excision theorem ([Bred97, Thm. 12.9]) the inclusion of pairs $\left(C \backslash C_{t^{\prime}}, C \backslash C_{t}\right) \rightarrow\left(X \backslash C_{t^{\prime}}, X \backslash C_{t}\right)$ induces an isomorphism

$$
\mathrm{H}^{*}\left(X \backslash C_{t^{\prime}}, X \backslash C_{t} ; G\right) \cong \mathrm{H}^{*}\left(C \backslash C_{t^{\prime}}, C \backslash C_{t} ; G\right) .
$$


The right-hand side is zero by Lemma 8.1 and the long cohomology sequence of the pair (see [Bred97, eqn. 24, p. 88]). So the left-hand side is also zero and therefore $\mathrm{H}^{*}\left(X \backslash C_{t} ; G\right) \rightarrow \mathrm{H}^{*}\left(X \backslash C_{t^{\prime}} ; G\right)$ is an isomorphism.

In the above theorem it is not necessary that $X$ is definably compact: it suffices that $C$ is bounded.

Remark 8.3. Let $R$ be the limit of a direct system $\left(R_{i} \mid f_{i, j}\right)_{i, j \in I}$ of Abelian groups and morphisms $f_{i, j}: R_{i} \rightarrow R_{j}$. Suppose that each $f_{i, j}$ is an isomorphism. Then for each $i$ the natural morphism $R_{i} \rightarrow R$ is an isomorphism.

Corollary 8.4. Suppose that $X$ is a definably compact set such that $C$ is a cell of maximal dimension of $X$ (for instance $X=C l(C)$ ). Then for every $t>0$ the inclusion map $X \backslash C \subset X \backslash C_{t}$ induces an isomorphism

$$
\mathrm{H}^{p}\left(X \backslash C_{t} ; G\right) \cong \mathrm{H}^{p}(X \backslash C ; G) .
$$

Proof. For $t>0$ we have

$$
\begin{aligned}
\mathrm{H}^{*}\left(X \backslash C_{t} ; G\right) & \cong \lim _{s} \mathrm{H}^{*}\left(X \backslash C_{s} ; G\right) \\
& \cong \mathrm{H}^{*}(X \backslash C ; G)
\end{aligned}
$$

where the first isomorphism follows from Lemma 8.2 and Remark 8.3, and the second one follows from the equality $X \backslash C=\bigcap_{s}\left(X \backslash C_{s}\right)$ and Corollary C.6.

Note that for $t$ big enough, $C_{t}$ is a singleton. So in particular, applying the theorem to $X=\bar{C}$, we have proved that there is a point $a \in C$ such that:

$$
\mathrm{H}^{p}(\bar{C} \backslash\{a\} ; G) \cong \mathrm{H}^{p}(\partial C ; G) .
$$

We do not know however whether $\partial C$ is a definable deformation retract of $\bar{C} \backslash\{a\}$.

Theorem 8.5. Let $X \subset M^{n}$ be a definably compact set and $G$ be an Abelian group. Then, for each $p, \mathrm{H}^{p}(X ; G)$ is finitely generated. Moreover $\mathrm{H}^{p}(X ; G)=0$ for $p>\operatorname{dim}(X)$.

Proof. By a Mayer-Vietoris argument. Decompose $X$ into cells. Let $C$ be a cell of $X$ of maximal dimension. Let $t>0$ be such that $C_{t} \neq \emptyset$ and write $X$ as the union of $X \backslash C_{t}$ and $C$. Consider the Mayer-Vietoris sequence (see [Bred97, eqn. 32, p. 98]) associated to this union:

$$
\ldots \rightarrow \mathrm{H}^{p-1}\left(C \backslash C_{t}\right) \rightarrow \mathrm{H}^{p}(X) \rightarrow \mathrm{H}^{p}\left(X \backslash C_{t}\right) \oplus \mathrm{H}^{p}(C) \rightarrow \mathrm{H}^{p}\left(C \backslash C_{t}\right) \rightarrow \ldots
$$


where we have omitted the coefficients $G$ in the notation. By Corollary 8.4 the inclusion $X \backslash C_{t} \subset X \backslash C$ induces an isomorphism in cohomology, so composing with this isomorphism we obtain

$$
\ldots \rightarrow \mathrm{H}^{p-1}\left(C \backslash C_{t}\right) \rightarrow \mathrm{H}^{p}(X) \rightarrow \mathrm{H}^{p}(X \backslash C) \oplus \mathrm{H}^{p}(C) \rightarrow \mathrm{H}^{p}\left(C \backslash C_{t}\right) \rightarrow \ldots
$$

Now $C$ has the cohomology of a point and $C \backslash C_{t}$ has the cohomology of an $(m-1)$-dimensional sphere. So the displayed part of the sequence above has the form:

$$
\begin{aligned}
& G \rightarrow \mathrm{H}^{p}(X) \rightarrow \mathrm{H}^{p}(X \backslash C) \oplus \mathrm{H}^{p}(C) \rightarrow 0, \\
& \text { or } \quad 0 \rightarrow \mathrm{H}^{p}(X) \rightarrow \mathrm{H}^{p}(X \backslash C) \oplus \mathrm{H}^{p}(C) \rightarrow G, \\
& \text { or } \quad 0 \rightarrow \mathrm{H}^{p}(X) \rightarrow \mathrm{H}^{p}(X \backslash C) \oplus \mathrm{H}^{p}(C) \rightarrow 0 ;
\end{aligned}
$$

where (8.6) applies for $p \in\{m, m-1\}$. By induction on the number of cells $\mathrm{H}^{p}(X \backslash C)$ is finitely generated, and vanishes for $p \geq m$. From the above sequences it then follows that the same holds for $\mathrm{H}^{p}(X)$.

\section{Elementary extensions and change of language}

Let $M$ be an o-minimal expansion of a group and let $X \subset M^{n}$ be a definable set. We have seen that we can associate to $X$ the spectral space $\widetilde{X}$ of all its types over $M$ (such a type can be identified with an ultrafilter of $M$ definable sets that contains $X$ ). The cohomology of $X$ has been defined as the cohomology of $\tilde{X}$. If $N \succ M$ is an elementary extension we may also associate to $X$ the spectral space $\widetilde{X(N)}$ (ultrafilters of $N$-definable sets that contains $X(N))$.

Theorem 9.1. Let $\theta: \widetilde{X(N)} \rightarrow \widetilde{X}$ be the map that sends a type over $N$ to its restriction over $M$. If $X$ is definably compact then $\theta$ induces an isomorphism $\mathrm{H}^{*}(\widetilde{X} ; G) \rightarrow \mathrm{H}^{*}(\widetilde{X(N)} ; G)$ for any Abelian group $G$.

Proof. For both $X$ and $X(N)$ we have an exact sequence as in equation (8.2) above. The terms of the two exact sequences so obtained are connected by the homomorphisms induced by $\theta$ in such a way that the resulting diagram commutes (it is important that in equation (8.2) we take the parameter $t$ in the small model $M$ ). The desired result then follows arguing as in Theorem 8.5 by induction on the number of cells.

We now extend the above result to certain type-definable sets. As above let $N \succ M$.

Theorem 9.2. Let $X \subset M^{n}$ be a definably compact set. Let $A \subset \tilde{X}$ be a type-definable closed subset and let $A(N):=\theta^{-1}(A) \subset \widehat{X(N)}$. Then $\theta$ 
induces an isomorphism $\mathrm{H}^{*}(A ; G) \rightarrow \mathrm{H}^{*}(A(N) ; G)$. In particular if $p$ is a closed type in $\widetilde{X}$, then the set $\theta^{-1}(p)$ of all types of $\widetilde{X(N)}$ which restrict to $p$ has the same cohomology of a point (so in particular it is connected).

Proof. Each closed type-definable set $A \subset \tilde{X}$ can be written as an intersection $\bigcap_{i \in I} X_{i}$ of definably compact sets $X_{i} \subset M^{n}$. Now observe that $A(N)=\bigcap_{i \in I} \widehat{X_{i}(N)}$. By Corollary C.6 and Theorem 9.1 $\mathrm{H}^{*}(A(N) ; G)=$ $\lim _{i \in I} \mathrm{H}^{*}\left(\widehat{X_{i}(N)} ; G\right)=\lim _{i \in I} \mathrm{H}^{*}\left(X_{i} ; G\right)=\mathrm{H}^{*}(A ; G)$.

In similar way we can prove:

Theorem 9.3. If $M_{1}$ is an o-minimal expansion of $M$ to a bigger language and $X \subset M^{n}$ is a definably compact set in $M$, then the map $\widetilde{X\left(M_{1}\right)} \rightarrow$ $\widetilde{X}$ sending each type in the language $L_{1}$ to its restriction to $L$ induces an isomorphism $\mathrm{H}^{*}(\widetilde{X} ; G) \rightarrow \mathrm{H}^{*}\left(\widetilde{X\left(M_{1}\right)} ; G\right)$ for any Abelian group $G$.

\section{Definable families in expansions of fields}

Definition 10.1. Given definable maps $f: X \rightarrow Y$ and $f^{\prime}: X^{\prime} \rightarrow Y^{\prime}$, we say that $f$ and $f^{\prime}$ have the same definable topological type iff there exist definable homeomorphisms $\lambda: X \rightarrow X^{\prime}$ and $\mu: Y \rightarrow Y^{\prime}$ making the following diagram commute:

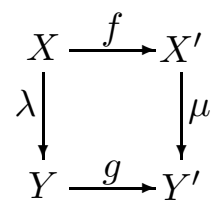

Definition 10.2. Let $\left(Y_{t}\right)_{t>0}$ be a family of subsets of $M^{n}$. We will say that $\left(Y_{t}\right)_{t>0}$ is decreasing if $Y_{t} \subseteq Y_{t^{\prime}}$ for every $t \leq t^{\prime}$.

If $M$ is an o-minimal expansion of a field, Corollary C.6 can be strengthened as follows.

Theorem 10.3. Assume that $M$ is an o-minimal expansion of a field. Let $\left(Y_{t}\right)_{t>0}$ be a definable decreasing family of definably compact subsets of some definable set $Y$. Let $A:=\bigcap_{t>0} Y_{t}$. Then for every sufficiently small $t$ we have a natural isomorphism induced by the inclusion

$$
\mathrm{H}^{*}(A ; \mathcal{F}) \cong \mathrm{H}^{*}\left(Y_{t} ; \mathcal{F}\right)
$$

for every sheaf $\mathcal{F}$ on $\tilde{Y}$. 
In the above theorem we cannot weaken the hypothesis to $Y_{t}$ closed (instead of definably compact). For instance, let $Y_{t}:=\left[\frac{1}{t}, \infty\right) \subseteq M$.

Note that Corollary 8.4 is a special case of Theorem 10.3. To prove the theorem, we need the following lemmata. Note that for the lemmata we do not need that $M$ expands a field, but only that it expands a group.

Lemma 10.4. Let $M$ be an o-minimal expansion of a group. Let $Y \subseteq$ $M^{n}$ be definable, and $f: Y \rightarrow M$ be a definable function (not necessarily continuous). Let $A \subseteq Y$ be the set of local minima of $f$. Then, $f(A)$ is finite.

Proof. If not, let $a<b \in M$ such that, for every $t \in(a, b)$ there exists $\gamma(t) \in Y$ such that $f(\gamma(t))=t$ and $\gamma(t)$ is a local minimum for $f$. By definable choice, we can assume that $\gamma$ is a definable continuous function. It follows that in any neighbourhood of $\gamma(t)$ there are points of the form $\gamma\left(t^{\prime}\right)$ with $t^{\prime}<t$. But $f\left(\gamma\left(t^{\prime}\right)\right)<f(\gamma(t))$, contradicting the fact that $\gamma(t)$ is a local minimum.

Lemma 10.5. Let $M$ be an o-minimal expansion of a group. Let $\left(Y_{t}\right)_{t>0}$ be a decreasing definable family of closed subsets of $M^{n}$. Then, there exists $t_{0}>0$ such that, for every $t \in M$ with $0<t<t_{0}$ we have

$$
Y_{t}=\bigcap_{t<u<t_{0}} Y_{u}
$$

Proof. Let $Y:=\bigcup_{t>0} Y_{t}$. Define

$$
\begin{gathered}
f: Y \rightarrow M^{\geq 0} \\
f(x):=\inf \left\{t: x \in Y_{t}\right\} .
\end{gathered}
$$

Define also $Z_{t}:=f^{-1}([0, t])$. Note that $Z_{t}=\bigcap_{t<u<t_{0}} Y_{u}$, and $Y_{t} \subseteq Z_{t}$. Therefore, the conclusion is equivalent to saying that, for every $0<t<t_{0}$, we have that $Y_{t}=Z_{t}$.

Claim 2. For every $t \geq 0$, if $x \in Z_{t} \backslash Y_{t}$, then $x$ is a local minimum for $f$.

If not, let $x \in Z_{t} \backslash Y_{t}$ such that $x$ is not a local minimum for $f$. Note that $f(x)=t$. Let $\gamma:(0, \varepsilon) \rightarrow Y$ be a definable function such that $\lim _{s \rightarrow 0} \gamma_{s}=x$ and $f\left(\gamma_{s}\right)<f(x)$. Then, $\gamma_{s} \in Y_{t}$. Since $Y_{t}$ is closed, we have that $x \in Y_{t}$, absurd.

By Lemma 10.4, there exists $t_{0}>0$ such that, for every $x \in Y$, if $0<f(x)<t_{0}$, then $x$ is not a local minimum for $f$. The claim implies the conclusion.

Remark 10.6. A few remarks about the above Lemma and its proof.

1. The function $f$ is lower semi-continuous, because $Z_{t}$ is closed for every $t$. 
2. The hypothesis that the $Y_{t}$ are closed is necessary.

3. Even if the function $f$ in the proof is continuous, we cannot conclude that $Y_{t}=Z_{t}$ always.

4. It is not true that there exists $t_{0}>0$ such that $f$ is continuous on $Y_{t_{0}}$.

Proof of Theorem 10.3. By Corollary C.6,

$$
\mathrm{H}^{*}(\widetilde{A} ; \mathcal{F})=\underset{t \rightarrow 0}{\lim _{t \rightarrow 0}} \mathrm{H}^{*}\left(\widetilde{Y}_{t} ; \mathcal{F}\right) .
$$

Since $\left(Y_{t}\right)_{t>0}$ is a definable family, and $M$ expands a field, the (definable) topological type of the $Y_{t}$ is eventually constant (this is a consequence of the trivialization theorem holding in o-minimal expansions of fields, see [Dries98]). Hence we can assume w.l.o.g. that the $Y_{t}$ are all definably homeomorphic, and therefore that their cohomology groups over $G$ are isomorphic to each other; let us denote by $P$ such group. Thus, for every $t \leq t^{\prime}$, the map induced by the inclusion

$$
\phi_{t}^{t^{\prime}}: \mathrm{H}^{*}\left(\widetilde{Y}_{t^{\prime}} ; \mathcal{F}\right) \rightarrow \mathrm{H}^{*}\left(\widetilde{Y}_{t} ; \mathcal{F}\right)
$$

is an endomorphism of $P$. It suffices to prove that $\phi_{t}^{t^{\prime}}$ is an isomorphism for all sufficiently small $t^{\prime}$ to prove the theorem. By Lemma 10.5, w.l.o.g. we can assume that, for every $t>0$,

$$
Y_{t}=\bigcap_{t^{\prime}>t} Y_{t^{\prime}}
$$

For convenience, define $\phi_{t}^{t^{\prime}}:=\phi_{t^{\prime}}^{t}$ if $t^{\prime} \leq t$, and $I:=M^{>0}$. Let $E$ be the equivalence relation on $I$ given by

$$
t E t^{\prime} \Leftrightarrow \phi_{t}^{t^{\prime}} \text { is an isomorphism. }
$$

Claim 3. E is a definable subset of $I^{2}$.

In fact, by the trivialization theorem, there exists a finite partition $\left\{C_{1}, \ldots, C_{n}\right\}$ of $\left\{\left(t, t^{\prime}\right) \in M^{2}: 0<t \leq t^{\prime}\right\}$ into definable sets, such that the definable topological type of the inclusion map $Y_{t} \rightarrow Y_{t^{\prime}}$ is constant on each $C_{k}$. Hence, the isomorphism type of the map $\phi_{t}^{t^{\prime}}$ is constant on each $C_{k}$, and thus $E$ is a finite union of some of the $C_{k}$.

Claim 4. For every $t \in I$ there exists $t_{0}>t$ such that, for every $t^{\prime} \in I$,

$$
t \leq t^{\prime}<t_{0} \Rightarrow t E t^{\prime}
$$


Since $Y_{t}=\bigcap_{t^{\prime}>t} Y_{t^{\prime}}$, by Corollary C.6 we have $\mathrm{H}^{*}\left(\widetilde{Y}_{t} ; \mathcal{F}\right)=\underset{t^{\prime} \rightarrow t^{+}}{\lim } \mathrm{H}^{*}\left(\widetilde{Y}_{t^{\prime}} ; \mathcal{F}\right)$;
namely,

$$
P=\underset{t^{\prime} \rightarrow t^{+}}{\lim }\left\{P, \phi_{t}^{t^{\prime}}\right\} .
$$

By definition of inductive limit, for every $p \in P$ there exists $t^{\prime}>t$ and $p^{\prime} \in P$ such that $p=\phi_{t}^{t^{\prime}}\left(p^{\prime}\right)$. However, $P$ is finitely generated, and therefore there exists $t_{0}>t$ such that $\phi_{t}^{t^{\prime}}$ is surjective for every $t_{0}>t^{\prime}>t$. Since $P$ is finitely generated, using Nakayama's lemma, we can conclude that $\phi_{t}^{t^{\prime}}$ is an isomorphism.

Claim 5. $I / E$ is finite

Claim 4 implies that each equivalence class of $E$ has non-empty interior. Since $E$ is definable, $I / E$ must be finite.

It follows from Claim 5 that there exists a left neighbourhood $J$ of 0 such that, for every $t, t^{\prime} \in J, t E t^{\prime}$. Therefore, for every $t \leq t^{\prime}$ in that neighbourhood $\phi_{t}^{t^{\prime}}$ is an isomorphism, and we conclude by Remark 8.3 .

Remark 10.7. In the situation of Theorem 10.3, let

$$
\begin{gathered}
f: Y \rightarrow M^{\geq 0} \\
f(x):=\inf \left\{t: x \in Y_{t}\right\} .
\end{gathered}
$$

We have remarked that $f$ might not be continuous. Assume that there exists $t_{0}>0$ such that $f$ is continuous on $Y_{t_{0}}$. Then, the proof of Theorem 10.3 can be simplified. In fact, by the trivialization theorem, we can assume that there exists a continuous definable map $\lambda: Y_{t_{0}} \backslash A \rightarrow F$ (where $F:=$ $\left.f^{-1}\left(t_{0}\right)\right)$, such that the map

$$
\mu:=(f, \lambda): Y_{t_{0}} \backslash A \rightarrow\left(0, t_{0}\right) \times F
$$

is a homeomorphism. Let $0<t \leq t^{\prime} \leq t_{0}$. Let $\theta:[0,1] \times\left[0, t^{\prime}\right] \rightarrow\left[0, t^{\prime}\right]$ be a definable strong deformation retraction between $\left[0, t^{\prime}\right]$ and $[0, t]$. Define

$$
\begin{aligned}
\Lambda:[0,1] \times Y_{t^{\prime}} & \rightarrow Y_{t^{\prime}} & & \\
\Lambda\left(s, \mu^{-1}(u, x)\right) & =\mu^{-1}(\theta(s, u), x) & & \text { if } 0<u<t^{\prime} \\
\Lambda(s, a) & =a & & \text { if } a \in A .
\end{aligned}
$$

Note that $\Lambda$ gives a definable strong deformation retraction between $Y_{t}^{\prime}$ and $Y_{t}$. Therefore the inclusion $Y_{t} \subseteq Y_{t^{\prime}}$ induces an isomorphism in cohomology.

The fact that in the situation of the above remark $Y_{t}$ is a deformation retract of $Y_{t^{\prime}}$ follows from a stronger results in [PetS07, Thm. A.5], where however it is assumed that 0 is a regular value for $f$.

Question 10.8. Under the hypothesis of Theorem 10.5, is there some $t_{0}>0$ such that $A$ is a deformation retract of $Y_{t}$ for every $0<t<t_{0}$ ? 
Note that in the proof of Theorem 10.3 we have used heavily the trivialization theorem, which holds only for expansions of fields.

Question 10.9. Can we weaken the hypothesis of Theorem 10.3 dropping the condition that $M$ expands a field (but saying instead that it expands a group)?

\section{Appendices}

\section{A Derived functors}

Let $\mathfrak{A}$ and $\mathfrak{B}$ be Abelian categories such that $\mathfrak{A}$ has enough injective objects (namely, every object of $\mathfrak{A}$ can be embedded in an injective one), and let $\Gamma: \mathfrak{A} \rightarrow \mathfrak{B}$ be an additive covariant left-exact functor.

In our applications, $\mathfrak{A}$ will be the category $\mathrm{Sh}_{X}$ of sheaves of Abelian groups on a fixed topological space $X, \mathfrak{B}$ the category of Abelian groups $\mathfrak{A} \mathfrak{b}$, and $\Gamma: \mathrm{Sh}_{X} \rightarrow \mathfrak{A} \mathfrak{b}$ the global section functor.

A chain complex in $\mathfrak{A}$ is a sequence of maps $C^{n} \stackrel{d^{n}}{\rightarrow} C^{n+1}$ in $\mathfrak{A}$ with $d^{n+1} \circ d^{n}=0$ for every $n \in \mathbb{Z}$. The chain complex $C^{*}=\left(C^{n}, d^{n}\right)_{n \in \mathbb{Z}}$ is exact if $\operatorname{Ker}\left(d^{n}\right)=\operatorname{Im}\left(d^{n-1}\right)$ for every $n$. A resolution of $A \in \mathfrak{A}$ is an exact chain complex of the form

$$
0 \rightarrow A \stackrel{j}{\rightarrow} I^{0} \stackrel{d^{0}}{\rightarrow} I^{1} \stackrel{d^{1}}{\rightarrow} I^{2}
$$

Given such a resolution, after applying $\Gamma$ we obtain a chain complex in $\mathfrak{B}$

$$
0 \rightarrow \Gamma(A) \stackrel{\Gamma(j)}{\rightarrow} \Gamma\left(I^{0}\right) \stackrel{\Gamma\left(d^{0}\right)}{\rightarrow} \Gamma\left(I^{1}\right) \stackrel{\Gamma\left(d^{1}\right)}{\rightarrow} \Gamma\left(I^{2}\right) \ldots
$$

which however need not be exact since $\Gamma$ is only left-exact. The lack of exactness is measured by the cohomology groups

$$
\mathrm{H}^{n}\left(\Gamma\left(I^{*}\right)\right)=\operatorname{Ker}\left(\Gamma\left(d^{n}\right)\right) / \operatorname{Im}\left(\Gamma\left(d^{n-1}\right)\right)
$$

where we stipulate that $d^{-1}:=0$. Note that $\mathrm{H}^{0}\left(\Gamma\left(I^{*}\right)\right)=\Gamma(A)$.

Now let us further assume that $0 \rightarrow A \rightarrow I^{*}$ is an injective resolution, namely a resolution such that each $I^{n}$ is an injective object of $\mathfrak{A}$. Then it turns out that $\mathrm{H}^{*}\left(\Gamma I^{*}\right)$ depends only on $A$ and not on the particular choice of the injective resolution. This can be seen as follows.

If $0 \rightarrow B \rightarrow C^{*}$ is another exact sequences in $\mathfrak{A}$ and $f: B \rightarrow A$ is a morphism in $\mathfrak{A}$, then (by injectivity of $I^{n}$ ) there exists a morphism of chain complexes $f^{*}: C^{*} \rightarrow I^{*}$ commuting with $f$ as in Diagram 1 below. 


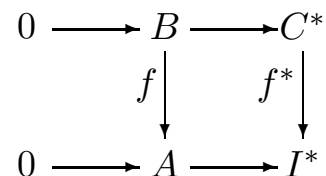

Diagram 1

Moreover $f^{*}$ is determined by $f$ up to chain homotopy (see Eisen95, Proposition A3.13]). Since $\Gamma$ preserves chain homotopies, we obtain a morphism (unique up to chain homotopy)

$$
\Gamma\left(f^{*}\right): \Gamma\left(C^{*}\right) \rightarrow \Gamma\left(I^{*}\right)
$$

which induces a unique map

$$
\mathrm{H}^{n}\left(\Gamma\left(f^{*}\right)\right): \mathrm{H}^{n}\left(\Gamma\left(C^{*}\right)\right) \rightarrow \mathrm{H}^{n}\left(\Gamma\left(I^{*}\right)\right) .
$$

It follows that if $0 \rightarrow A \rightarrow I^{*}$ and $0 \rightarrow A \rightarrow C^{*}$ are two injective resolutions of $A \in \mathfrak{A}$, then the identity morphism $\operatorname{id}_{A}$ on $A$ induces a canonical isomorphism

$$
\mathrm{H}^{n}\left(\Gamma\left(\mathrm{id}_{A}\right)\right): \mathrm{H}^{n}\left(\Gamma\left(I^{*}\right)\right) \cong \mathrm{H}^{n}\left(\Gamma\left(C^{*}\right)\right) .
$$

The right derived functor $\mathrm{R}^{n} \Gamma: \mathfrak{A} \rightarrow \mathfrak{B}$ can thus be defined, up to a canonical isomorphism, by:

$$
\mathrm{R}^{n} \Gamma(A):=\mathrm{H}^{n}\left(\Gamma I^{*}\right)
$$

If $f$ is as in the Diagram 1 and all the $I^{n}, C^{n}$ are acyclic, we define

$$
\mathrm{R}^{n} \Gamma(f):=\mathrm{H}^{n}\left(\Gamma f^{*}\right)
$$

An object $C \in \mathfrak{A}$ is acyclic (for $\Gamma$ ), if $\mathrm{R}^{n} \Gamma(C)=0$ for all $n>0$. Any injective object in $\mathfrak{A}$ is acyclic for any additive functor. If $A \in \mathfrak{A}$ and $0 \rightarrow A \rightarrow C^{*}$ is an acyclic resolution of $A$, then the natural map $\mathrm{H}^{n}\left(\Gamma\left(C^{*}\right)\right) \rightarrow \mathrm{R}^{n} \Gamma(A)$ is an isomorphism (the proof in Bred97, §II.4] works in this context). So we can compute $\mathrm{R}^{n} \Gamma(A)$ using any acyclic resolution of $A$, not necessarily injective. We have the following sufficient condition for acyclicity:

Lemma A.1. Let $\mathfrak{C}$ be a class of objects in $\mathfrak{A}$. Assume that

1. All injective objects of $\mathfrak{A}$ are in $\mathfrak{C}$;

2. If $0 \rightarrow C^{\prime} \rightarrow A \rightarrow A^{\prime \prime} \rightarrow 0$ is a short exact sequence (in $\mathfrak{A}$ ), with $C^{\prime} \in \mathfrak{C}$, then

$$
0 \rightarrow \Gamma\left(C^{\prime}\right) \rightarrow \Gamma(A) \rightarrow \Gamma\left(A^{\prime \prime}\right) \rightarrow 0
$$

is a short exact sequence (in $\mathfrak{B})$ : 円

\footnotetext{
${ }^{1}$ Note that, since $\Gamma$ is left-exact, $0 \rightarrow \Gamma\left(C^{\prime}\right) \rightarrow \Gamma(A) \rightarrow \Gamma\left(A^{\prime \prime}\right)$ is always exact.
} 
3. If $0 \rightarrow C^{\prime} \rightarrow C \rightarrow A^{\prime \prime} \rightarrow 0$ is a short exact sequence, with $C^{\prime}, C \in \mathfrak{C}$, then $A^{\prime \prime} \in \mathfrak{C}$.

Then,

(a) If $0 \rightarrow C^{-1} \rightarrow C^{0} \rightarrow C^{1} \ldots$ is an exact sequence in $\mathfrak{C}$, then $0 \rightarrow$ $\Gamma\left(C^{-1}\right) \rightarrow \Gamma\left(C^{0}\right) \rightarrow \Gamma\left(C^{1}\right) \ldots$ is also exact;

(b) $\mathrm{R}^{n} \Gamma(C)=0$ for every $C \in \mathfrak{C}$ and $n>0$ (namely, all objects in $\mathfrak{C}$ are acyclic).

Proof. Point (b) follows from (a). The proof of (a) is in God73, Thm. II.3.1.3] for the class $\mathfrak{C}$ of flabby sheaves: it generalizes easily to this context.

\section{B Flabby and soft sheaves}

Let $X$ be an arbitrary topological space (not necessarily Hausdorff). We shall give some results about sheaf cohomology on $X$; for unproved facts in this and the following sections, the reader can look either in Bred97 or in God73.

Definition B.1. We shall denote by $\mathrm{Sh}_{\mathrm{X}}$ the category of sheaves of Abelian groups on $X$. Sh $\mathrm{X}$ is an Abelian category, with enough injective objects. Let

$$
\begin{aligned}
\Gamma: \mathrm{Sh}_{\mathrm{X}} & \rightarrow \mathfrak{A b} \\
\mathcal{F} & \mapsto \mathcal{F}(X)
\end{aligned}
$$

be the global section functor. It is well-known that $\Gamma$ is left-exact. The $n$-th cohomology functor on $X$ is the $n$-th right derived functor of $\Gamma$

$$
\mathrm{H}^{n}(X ; \mathcal{F}):=\mathrm{R}^{n} \Gamma(\mathcal{F}) .
$$

Given $A \subseteq X$ we shall write $\mathrm{H}^{*}(A ; \mathcal{F})$ for $\mathrm{H}^{*}(A ; \mathcal{F}\lceil A)$.

A sheaf on $X$ is called flabby if for every open subset $A$ of $X$ any section of $\mathcal{F}$ on $A$ can be extended to the whole of $X$. Any injective sheaf is flabby, and any flabby sheaf is acyclic. A sheaf $\mathcal{F}$ on $X$ is soft if for every closed subset $A$ of $X$ any section of $\mathcal{F}$ on $A$ can be extended to the whole of $X$. On Hausdorff paracompact spaces, soft sheaves are flabby, and therefore acyclic.

Many results on sheaf cohomology are stated in [God73] or Bred97 for paracompact Hausdorff spaces, but an analysis of the proofs shows that they hold more generally for arbitrary $\mathrm{PcN}$ spaces (cf. \$2).

Lemma B.2. Let $X$ be a topological space and let $A \subseteq X$ have a basis of PcN neighbourhoods. Let $\mathcal{F} \in \mathrm{Sh}_{\mathrm{X}}$, and $s$ be a section of $\mathcal{F}$ on $A$. Then, $s$ can be extended to a neighbourhood of $A$. Therefore,

$$
\mathcal{F}(A)=\lim _{\overrightarrow{A \subseteq U}} \mathcal{F}(U),
$$

using the neighbourhoods $U$ of A, ordered by reversed inclusion. 
Proof. The proof of God73, Thm. 3.3.1, p. 150] works under our hypothesis.

Lemma B.3. Let $\mathcal{F}$ be a flabby sheaf on $X$. If $X$ is PcN, then $\mathcal{F}$ is soft. More generally, if $X$ is an arbitrary topological space, $\mathcal{F}$ is a flabby sheaf on $X$, and $A$ is a subspace of $X$ with a basis of PcN neighbourhoods, then $\mathcal{F}$ induces a soft sheaf on $A$.

Proof. Let $C \subseteq A$ be closed in $A$, and $s$ be a section of $\mathcal{F}$ on $C$. By Lemma B.2, $s$ can be extended to a neighbourhood $W$ of $C$ in $X$. Since $\mathcal{F}$ is flabby, $s$ can be further extended to $X$, and a fortiori to $A$.

Lemma B.4. If $X$ is a topological space and $A \subseteq X$ has a basis of PcN neighbourhoods in $X$, then $A$ is PcN. More precisely, if $A \subseteq X$ has a basis of normal neighbourhoods, then $A$ is normal. If $A$ has a basis of paracompact neighbourhoods, then $A$ is paracompact.

Proof. Normality: First note that a space is normal if and only if every open covering consisting of two open sets $U, V$ has a shrinking. Given an open covering $\{U, V\}$ of $A$, let $U^{\prime}, V^{\prime}$ be open sets of $X$ with $U^{\prime} \cap A=U$ and $V^{\prime} \cap A=V$. Let $W$ be a normal open neighbourhood of $A$ contained in $U^{\prime} \cup V^{\prime}$. We can assume $W=U^{\prime} \cup V^{\prime}$. By the normality of $W$ we can find a shrinking $\left\{U_{0}^{\prime}, V_{0}^{\prime}\right\}$ of $\left\{U^{\prime}, V^{\prime}\right\}$. The intersections of $U_{0}^{\prime}$ and $V_{0}^{\prime}$ with $A$ is a shrinking of $\{U, V\}$. The proof of paracompactness is similar.

Theorem B.5. Assume that $X$ is a PcN space. Then any soft sheaf on $X$ is acyclic.

Proof. (See God73, §II-3.5].) Lemma A.1 gives a sufficient condition for acyclicity, so it suffices to verify its three hypothesis.

Point 1. We need to show that any injective sheaf on $X$ is soft. This follows from the fact that any injective sheaf is flabby, and any flabby sheaf on a $\mathrm{PcN}$ space is soft (Lemma B.3).

Point 2. This is proved in God73, Thm. II-3.5.2, p. 153] for Hausdorff paracompact spaces (or supports), but the proof works also for $\mathrm{PcN}$ spaces.

Point 3. Assume that $0 \rightarrow \mathcal{F}^{\prime} \rightarrow \mathcal{F} \rightarrow \mathcal{F}^{\prime \prime} \rightarrow 0$ is an exact sequence in $\mathrm{Sh}_{\mathrm{X}}$, with $\mathcal{F}^{\prime}$ and $\mathcal{F}$ soft. We have to prove that $\mathcal{F}^{\prime \prime}$ is also soft. Let $C \subseteq X$ be closed. By the second point, since $\mathcal{F}^{\prime}\lceil C$ is soft,

$$
\mathcal{F}(C) \rightarrow \mathcal{F}^{\prime \prime}(C)
$$

is surjective. Hence, a section $s^{\prime \prime}$ of $\mathcal{F}^{\prime \prime}$ on $C$ is represented by a section $s$ of $\mathcal{F}$ on $C$. Since $\mathcal{F}$ is soft, $s$ can be extended to all $X$, and hence also $s^{\prime \prime}$ can be extended to $X$. 


\section{Taut subspaces}

Definition C.1. Let $A \subseteq X$, and $\mathcal{F} \in \mathrm{Sh}_{\mathrm{X}}$. For every neighbourhood $U$ of $A$ in $X$, the inclusion map $\lambda_{U}^{A}: A \rightarrow U$ induces a map in cohomology $\mathrm{H}^{*}\left(\lambda_{U}^{A}\right): \mathrm{H}^{*}(X ; \mathcal{F}) \rightarrow \mathrm{H}^{*}(A ; \mathcal{F})$. If $U \subseteq V$, the maps $\mathrm{H}^{*}\left(\lambda_{V}^{U}\right), \mathrm{H}^{*}\left(\lambda_{U}^{A}\right)$, and $\mathrm{H}^{*}\left(\lambda_{V}^{A}\right)$ commute. Hence, we have a canonical map

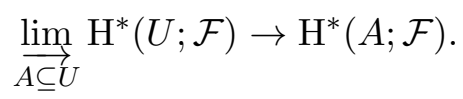

We say that $A$ is taut in $X$ for the sheaf $\mathcal{F}$ if the above map is an isomorphism. We say that $A$ is taut in $X$ iff $A$ is taut for every sheaf $\mathcal{F}$.

We give an example of a normal PcN space $X$ with a quasi-compact subspace $A$ which is not taut in $X$.

Example C.2. Let $X=\{a, b, c, d\}$ partially ordered so that $a=\min X, d=$ $\max X$ and $b, c$ are incomparable. Put the following topology on $X$ : a set is closed iff it is downward closed in this order. Then $X$ is a normal spectral space. The subset $A=\{b, c\}$ of $X$ is quasi-compact (since it is finite) but not taut. In fact $V=\{b, c, d\}$ is the smallest open set containing $A$, but $V$ is connected and $A$ is disconnected. Hence

$$
\mathrm{H}^{0}(A, \mathbb{Z})=\mathbb{Z}^{2} \neq \mathbb{Z}=\mathrm{H}^{0}(V, \mathbb{Z})=\underset{\overrightarrow{A \subseteq U}}{\lim _{\longrightarrow}} \mathrm{H}^{0}(U, \mathbb{Z}) .
$$

The above example shows that if we want to prove that a certain subspace is taut, we need some normality assumptions on its neighbourhoods.

Lemma C.3. Let $A \subseteq X$, and $\left(Y_{i}\right)_{i \in I}$ be a family of subsets of $X$ indexed by a filtered set $I$, such that $\left(Y_{i}\right)_{i \in I}$ is decreasing, and $A \subseteq \bigcap_{i \in I} Y_{i}$. Assume that $A$ and each $Y_{i}$ are taut in $X$, and that, for every neighbourhood $V$ of $A$, there exists $i \in I$, such that $Y_{i} \subseteq V$. Then for every $\mathcal{F} \in \mathrm{Sh}_{\mathrm{X}}$,

$$
\mathrm{H}^{*}(A ; \mathcal{F})=\underset{i \in I}{\lim _{i \in I}} \mathrm{H}^{*}\left(Y_{i} ; \mathcal{F}\right)
$$

Proof. Since $A$ and each $Y_{i}$ are taut in $X$,

$$
\mathrm{H}^{*}(A ; \mathcal{F})=\lim _{\overrightarrow{A \subseteq U}} \mathrm{H}^{*}(U ; \mathcal{F})=\lim _{i \in I} \lim _{Y_{i} \subseteq U} \mathrm{H}^{*}(U ; \mathcal{F})=\lim _{i \in I} \mathrm{H}^{*}\left(Y_{i} ; \mathcal{F}\right) .
$$

Theorem C.4. Let $X$ be an arbitrary topological space and let $A \subseteq X$. Assume that $A$ has a basis of PcN neighbourhoods in $X$. Then $A$ is taut in $X$.

Proof. (See God73, Thm. II.4.11.1].) Let $\mathcal{F} \in \mathrm{Sh}_{\mathrm{X}}$, and $0 \rightarrow \mathcal{F} \rightarrow \mathcal{C}^{*}$ be an injective resolution (in $\mathrm{Sh}_{\mathrm{X}}$ ). By Lemma B.2, $\mathcal{C}^{*}(A)=\underline{\lim }_{A \in U} \mathcal{C}^{*}(U)$. Since any injective sheaf is flabby (see Bred97]), by Lemma B.3,

$$
0 \rightarrow \mathcal{F}\left\lceil A \rightarrow \mathcal{C}^{*} \uparrow A\right.
$$


is a soft resolution in $\mathrm{Sh}_{A}$. Moreover $A$ is $\mathrm{PcN}$ (Lemma B.4), so any soft sheaf on $A$ is acyclic (Lemma B.5) and therefore the cohomology of $\mathcal{F}\lceil A$ can be computed by soft resolutions. So we obtain:

$$
\mathrm{H}^{p}\left(A ; \mathcal{F}\lceil A)=\mathrm{H}^{p}\left(\Gamma_{A}\left(\mathcal{C}^{*}\lceil A)\right)=\mathrm{H}^{p}\left(\lim _{\overrightarrow{A \subseteq U}} \mathcal{C}^{*}(U)\right)=\lim _{\overrightarrow{A \subseteq U}} \mathrm{H}^{p}(U ; \mathcal{F}) .\right.\right.
$$

where the last equality follows from the fact that the homology functor from complexes of modules over a fixed ring $R$, to $R$-modules, preserves inductive limits over filtered sets (see God73, $\S I .2 .1]$ ).

Corollary C.5. (Delfs85, Jones06, EdmJP05) Let $X$ be a definable set in an o-minimal expansion of a group. Let $A$ be a quasi-compact subset of $\widetilde{X}$ (this assumption holds in particular if $A$ is definable, or type-definable). Then $A$ is taut in $\tilde{X}$.

Corollary C.6. Let $\left(Y_{t}\right)_{t>0}$ be a definable family of definably compact subsets of some definable set $Y$, such that $Y_{t^{\prime}} \subseteq Y_{t}$ for every $0<t^{\prime}<t$. Let $A:=\bigcap_{t>0} Y_{t}$. Then the inclusion induces an isomorphism

$$
\mathrm{H}^{*}(A ; \mathcal{F}) \cong \underset{t \rightarrow 0}{\lim _{t \rightarrow 0}} \mathrm{H}^{*}\left(Y_{t} ; \mathcal{F}\right)
$$

for every sheaf $\mathcal{F}$ on $\tilde{Y}$.

Proof. Since the $Y_{t}$ are definably compact, for every definable neighbourhood $U$ of $A$ there exists $t>0$ such that $Y_{t} \subseteq U$. Moreover $A$ and $Y_{t}$ are taut by Theorem C.4 or Corollary C.5. Hence we can apply Lemma C.3

\section{D Čech cohomology}

Definition D.1. For every open covering $\mathcal{U}$ of $X$ and sheaf $\mathcal{F}$ on $X$, we can define the Cech cohomology groups $\check{\mathrm{H}}^{*}(\mathcal{U} ; \mathcal{F})$ as in Bred97. If $\mathcal{V}$ is a refinement of $\mathcal{U}$, there exists a canonical map $\check{\mathrm{H}}^{*}(\mathcal{U} ; \mathcal{F}) \rightarrow \mathrm{H}^{*}(\mathcal{V} ; \mathcal{F})$. We remind that the Cech cohomology groups of $X$ are defined as $\check{\mathrm{H}}^{*}(X ; \mathcal{F}):=$ $\lim _{\mathcal{U}} \check{\mathrm{H}}(\mathcal{U} ; \mathcal{F})$.

Lemma D.2. Let $X$ be a PcN space and $\mathcal{F}$ be a presheaf on $X$, such that $\hat{\mathcal{F}}=0$, where $\hat{\mathcal{F}}$ is the sheaf generated by $\mathcal{F}$. Then, $\check{\mathrm{H}}^{*}(X ; \mathcal{F})=0$.

Proof. Bredon [Bred97, Thm. III.4.4], Godement [God73, Thm. II.5.10.2] and Spanier Span81, Thm. 6.7.16] prove the above lemma for the case when $X$ is Hausdorff and paracompact. Their proofs work also for $X \mathrm{PcN}$, without need of modifications.

Lemma D.3. Let $X$ be a PcN space, and $\mathcal{F}$ be a presheaf on $X$. Denote by $\hat{\mathcal{F}}$ the sheaf generated by $\mathcal{F}$. Then, the canonical map $\theta: \mathcal{F} \rightarrow \hat{\mathcal{F}}$ induces an isomorphism

$$
\check{\mathrm{H}}^{*}(X ; \mathcal{F}) \stackrel{\cong}{\longrightarrow} \check{\mathrm{H}}^{*}(X ; \hat{\mathcal{F}}) .
$$


Lemma D.4. Let $X$ be a PcN space, and $\mathcal{F}$ be a sheaf on it. Then, there is a natural isomorphism

$$
\check{\mathrm{H}}^{*}(X ; \mathcal{F}) \cong \mathrm{H}^{*}(X ; \mathcal{F}) .
$$

Proof of Lemmata D.3 and D.4. Bredon Bred97, Cor. III.4.5, Cor. III.4.12] and Godement God73, Cor. II.5.10] prove the above lemmata for the case when $X$ is Hausdorff and paracompact. The proof for the $\mathrm{PcN}$ case can be done as in [Bred97]. The main ingredient is Lemma D.2; the rest are algebraic manipulations that do not use any property of the space.

In the case of $X$ normal spectral space, Lemma D.4 was already proven in [CarrC83].

\section{References}

[Bred97] G. E. Bredon. Sheaf theory. Graduate Texts in Mathematics (Vol. 170), Second edition. Springer-Verlag, New York, 1997. xii+502 pp.

[CarrC83] M. Carral, M. Coste. Normal spectral spaces and their dimension, J. Pure Appl. Algebra 30 (1983) 227-235

[CosteR82] M. Coste, M.-F. Roy, La topologie du Spectre Reel, in: D. W. Dubois and T. Recio, eds., Ordered Fields and Real Algebraic Geometry, Contemporary Mathematics 8, 1982, pp. 27-59

[Delfs85] H. Delfs. The homotopy axiom in semialgebraic cohomology. Journal für die reine und angewandte Mathematik 355 (1985) $108-128$

[Dries98] L. van den Dries. Tame Topology and o-minimal structures. London Math. Soc. Lecture Notes Series, vol. 248. Cambridge Univ. Press 1998. x+180 pp.

[God73] Roger Godement. Topologie algébrique et théorie des faisceaux. Troisime édition. Publications de l'Institut de Mathèmatique de l'Université de Strasbourg, XIII. Actualitś Scientifiques et Industrielles, No. 1252. Hermann, Paris, 1973. viii+283 pp.

[EdmJP05] M. Edmundo, G. O. Jones, N. J. Peatfield, Sheaf cohomology in o-minimal structures, Journal of Mathematical Logic, Vol. 6, No. 2 (2006) 163179

[EilS52] S. Eilenberg, N. Steenrod. Foundations of algebraic topology. Princeton University Press, Princeton, New Jersey, 1952. $\mathrm{xv}+328 \mathrm{pp}$. 
[Eisen95] D. Eisenbud. Commutative algebra, With a view toward algebraic geometry. Graduate Texts in Mathematics (vol. 150). Springer-Verlag, New York, 1995.

[Eng89] R. Engelking. General topology. Rev. and compl. ed. (English) Sigma Series in Pure Mathematics, 6. Berlin: Heldermann Verlag. viii, 529 p.

[Jones06] G. O. Jones. Local to global methods in o-minimal expansions of fields, PhD thesis, Oxford 2006, pp. 83

[PetSS00] Y. Peterzil, P. Speissegger, S. Starchenko. Adding multiplication to an o-minimal expansion of the additive group of real numbers. Logic Colloquium '98 (Prague), Lecture Notes in Logic 13, 357362. Assoc. Symbol. Logic, Urbana, IL, 2000.

[PetS07] Y. Peterzil, S. Starchenko. Computing o-minimal topological invariants using differential topology. Trans. Amer. Math. Soc. 359 (2007), no. $3,1375-1401$

[Pillay87] A. Pillay. First order topological structures and theories. The Journal of Symbolic Logic, vol. 52, n. 3 (1987) 763 - 778

[Pillay88] A. Pillay, Sheaves of continuous definable functions. The Journal of Symbolic Logic, vol. 53, n. 4 (1988) 1165 - 1169

[Span81] E. H. Spanier. Algebraic topology. Springer-Verlag, New York, 1981. Corrected reprint of the 1966 original.

[Wallm38] H. Wallman. Lattices and Topological Spaces. Annals of Mathematics (2) 39 (1938) 112-126

Università di Pisa, Dipartimento di Matematica, Largo Bruno Pontecorvo 5, 56127 PisA, ItAly. E-mail: berardu@dm.unipi.it.

Università di Pisa, Dipartimento di Matematica, Largo Bruno Pontecorvo 5, 56127 PISA, ITALy. E-mail: fornasiero@dm.mail.unipi.it. 